
Informations

une revue Gallia

Rhône-Alpes | 2008

\title{
Lyon
}

Réseau souterain des "arrêtes de poisson"

\section{Emmanuel Bernot}

\section{(2) OpenEdition \\ 12 Journals}

Édition électronique

URL : http://journals.openedition.org/adlfi/1818

ISSN : 2114-0502

Éditeur

Ministère de la culture

Référence électronique

Emmanuel Bernot, « Lyon », ADLFI. Archéologie de la France - Informations [En ligne], Rhône-Alpes, mis en ligne le 01 mars 2008, consulté le 22 avril 2019. URL : http://journals.openedition.org/adlfi/1818

Ce document a été généré automatiquement le 22 avril 2019.

(c) Ministère de la Culture et de la Communication, CNRS 


\section{Lyon}

Réseau souterain des "arrêtes de poisson"

\section{Emmanuel Bernot}

\section{Identifiant de l'opération archéologique : 9856}

Date de l'opération : 2008 (EV)

1 Le diagnostic archéologique mené au cours des mois de juin et juillet 2008 à l'intérieur des galeries souterraines de la Balme Saint-Clair a été réalisé dans le cadre du projet de doublement du tunnel de la Croix-Rousse (Lyon, $1^{\mathrm{er}}$ ). Il a permis de mettre au jour un vaste réseau militaire aux multiples fonctions.

2 Le réseau de galeries en arêtes de poisson se développe d'est en ouest, du Rhône à la rue Magneval. Il s'articule autour d'une galerie principale (la colonne vertébrale), longue d'au moins $156 \mathrm{~m}$ et marquée par trois coudes successifs, de part et d'autre de laquelle se déploient trente-deux galeries, organisées par paires (les arêtes). Chacune de ces arêtes, longue d'une trentaine de mètres et terminée en cul-de-sac, est reliée à la colonne vertébrale par un puits carré. À l'origine, ces seize puits remontaient à la surface et descendaient aussi vers une seconde colonne vertébrale, construite à l'aplomb de la première, dont elle reprend le plan sans cependant desservir d'arête.

3 Toutes ces galeries sont à l'origine maçonnées et présentent une homogénéité de construction absolue, tant dans leurs gabarits que dans les matériaux mis en oeuvre; seules les hauteurs peuvent varier d'une arête à l'autre. À l'exception de quelques grès provenant du substrat local, les maçonneries sont composées de calcaire finement cristallin de couleur beigeâtre à rosâtre (calcaire de l'Aalénien). Les finitions apportées aux galeries ainsi qu'aux puits sont similaires d'un bout à l'autre du réseau. Ainsi, les joints beurrés de leurs parois ont systématiquement été soulignés à la pointe de la truelle et un mortier de finition a été appliqué à la jonction des parois et des retombées de voûte. Ce travail semble avoir été en partie réalisé par des enfants comme en témoignent plusieurs empreintes de mains et de très nombreuses traces de doigts, 
formant parfois des dessins, laissées dans le mortier frais (Fig. $\mathrm{n}^{\circ} 1$ : Dessins faits au doigt dans le mortier de finition des galeries). Enfin, le sol des galeries est formé d'un radier en hérisson recouvert d'une chape de mortier de bonne tenue. Le radier empêche la formation de poches d'eau à la base des murs; il en garantit ainsi la bonne conservation dans le temps. La chape et le hérisson permettent de stabiliser les parois des galeries qui ne sont généralement pas fondées plus profondément que la base des radiers.

4 Au total, pour le réseau en arêtes de poisson, plus de 1,4 km de galeries ont été creusés et maçonnés: environ $960 \mathrm{~m}$ de galeries en arêtes, $312 \mathrm{~m}$ de colonnes vertébrales et environ $144 \mathrm{~m}$ pour les galeries situées sous la rive du fleuve. À cela, il faut ajouter les seize puits qui, à eux seuls, représentent une longueur cumulée estimée à près de $480 \mathrm{~m}$.

5 Au nord, plusieurs autres galeries (les antennes), s'inscrivent dans la continuité de cet ouvrage. Elles sont connectées aux arêtes de poisson par l'intermédiaire d'une galerie de $123 \mathrm{~m}$ de long et se raccordant à l'un des puits de l'antenne sud.

6 L'extension septentrionale du réseau est formée de deux galeries linéaires, dont l'extrémité est localisée à proximité de la rue des Fantasques. L'antenne nord, qui peut être restituée sur une longueur d'au moins $215 \mathrm{~m}$, s'étend actuellement au-delà de l'église Saint-Bernard. L'antenne méridionale est pour sa part conservée sur $296 \mathrm{~m}$, ce qui situe son extrémité occidentale sous la rue Vaucanson. On trouve plus au sud, une troisième galerie longue de $12,8 \mathrm{~m}$ et parallèle aux deux antennes. Ces trois galeries parallèles, orientées d'est en ouest, sont distantes les unes des autres de $12 \mathrm{~m}$ à $13 \mathrm{~m}$ et ont été percées à des altitudes différentes. Enfin, elles présentent toutes les trois un pendage d'est en ouest, conforme au relief de la colline, avec un dénivelé important sous le versant qui s'adoucit nettement sous le plateau de la Croix-Rousse.

7 Ces galeries ont une architecture en tout point comparable à celle des arêtes de poisson. Elles sont composées de tronçons horizontaux, mis bout à bout, en contrebas les uns des autres, afin de donner à l'ensemble le dénivelé souhaité. Ces tronçons qui sont, à l'origine, symétriques d'une antenne à l'autre, sont raccordés les uns aux autres par des décrochements verticaux du sol et de la voûte, qui correspondent aux parois d'un puits débouchant probablement à la surface, comme dans les arêtes. On peut sur ce principe restituer vingt-trois puits au-dessus de l'antenne sud, dont dix sont attestés, et onze audessus de l'antenne nord.

Plusieurs salles voûtées ont été construites, à intervalle variable, au-dessus des antennes. Elles semblent bâties sur le même plan, bien visible pour deux d'entre-elles qui sont presque intégralement conservées. Près de onze salles peuvent être restituées grâce aux observations de terrain, aux plans anciens et aux documents d'archives. Neuf d'entre elles relient les antennes nord et sud; les deux autres mettent en relation l'antenne méridionale et le petit tronçon de galerie situé plus au sud. On accède à ces salles par l'intermédiaire d'une ouverture aménagée dans la voûte de l'antenne nord, située en contrebas. De ce côté, une seconde trémie rectangulaire est également percée dans le berceau de chaque salle. Elle est surmontée d'un puits de plan carré bâti sur l'extrados de la voûte et remontant vraisemblablement à la surface. A l'opposé, la communication entre la salle et l'antenne sud, située près de $9 \mathrm{~m}$ en contrebas, se fait par un puits. Enfin, les galeries et les salles constituant l'extension septentrionale du réseau présentent des maçonneries similaires à celles des arêtes de poisson, tant dans leurs gabarits que dans leur mode de construction. 
9 L'extension nord du réseau s'étend sur une longueur d'au moins $300 \mathrm{~m}$ et comporte, dans son état actuel de dégagement, plus de $520 \mathrm{~m}$ de galeries. Leur étendue ne nous est cependant pas intégralement connue.

10 Le percement des galeries du réseau s'est opéré à partir des puits. Ces derniers ont d'abord servi de repères topographiques, en permettant de projeter sous terre le plan qu'ils dessinaient en surface. Seule la galerie qui relie les antennes au réseau des arêtes, paraît en avoir été dépourvue, ce qui constitue en soi une prouesse technique. Les puits ont ensuite été utilisés pour extraire les matériaux issus du creusement des galeries (constitués pour l'essentiel de sables molassiques), comme pour y acheminer les matériaux de construction. Sans doute ont-ils aussi servi d'accès pour les nombreux ouvriers que le chantier a dû mobiliser. Ils paraissent en revanche insuffisants pour avoir, à eux seuls, apportés l'éclairage nécessaires à ces travaux.

11 L'ensemble du réseau souterrain formé par les arêtes de poisson et son extension nord fut édifié d'un seul tenant. Toutefois ce chantier semble avoir été brutalement stoppé sans être en tout point achevé. De plus, la découverte d'une galerie non maçonnée à l'ouest des arêtes de poisson, comportant deux départs en vis-à-vis, paraît témoigner d'une extension avortée. Enfin, les galeries ne semblent jamais avoir réellement servi. On n'y trouve ni trace d'utilisation, ni marque d'aménagement dans les maçonneries ou au sol pouvant témoigner de la présence de structures légères.

12 L'homogénéité de la maçonnerie comme l'absence de trace de reprise montrent que le réseau en arêtes de poisson forme un ensemble architectural cohérent qui, de la rive du Rhône au plateau de la Croix-Rousse, relève d'une seule et même campagne de construction. Dans l'état actuel de la recherche, tous les éléments concordent pour faire de ce réseau un accessoire de la citadelle royale de Lyon (citadelle Saint-Sébastien), construite en 1564 sur la plateau de la Croix-Rousse, sur l'ordre de Charles IX et démantelée à la demande et au frais de la ville en 1585 .

Enfin, la fonction du réseau en arêtes de poisson peut être déterminée au regard de son architecture et du contexte dans lequel elle fut élaborée. Pris dans sa globalité, le réseau s'articule autour d'une galerie principale qui met en communication la citadelle SaintSébastien et le Rhône.

Cette galerie de circulation a deux fonctions :

- permettre d'accéder à la forteresse à l'insu de la population lyonnaise contre laquelle elle est tournée, d'une part ;

16 - et desservir de l'autre, par l'intermédiaire de puits, les deux zones de stockage qui se développent à un niveau supérieur : les arêtes de poisson (stricto sensu)et les salles de la partie nord du réseau.

17 Chacun de ces entrepôts souterrains est en outre desservi par une galerie qui lui est propre.

18 Leur approvisionnement pouvait se faire depuis le Rhône, dont le niveau des hautes eaux était nécessairement inférieur à l'étiage actuel. Il pouvait également s'effectuer par les puits situés à l'entrée des arêtes ou par les trémies aménagées dans les voûtes des salles, quoique ces dispositifs, indispensables à la construction, avaient peut-être aussi pour fonction de ventiler le réseau. Cependant, même dotés d'emmarchements et d'échelles de bois, les multiples paliers imposés par le dénivelé de plus de $70 \mathrm{~m}$ qui séparait le Rhône de la citadelle rendaient sans doute la circulation intérieure très incommode, surtout dans 
l'obscurité : la faible lumière dispensée par les puits étant arrêtée par les platelages qui permettaient leur franchissement au niveau supérieur. Ces difficultés restreignaient donc l'usage des magasins au stockage de matériel de peu de poids et de faible volume: vivres, munitions et armement léger à l'usage des troupes de la citadelle.

\section{ANNEXES}

Fig. $n^{\circ} 1$ : Dessins faits au doigt dans le mortier de finition des galeries

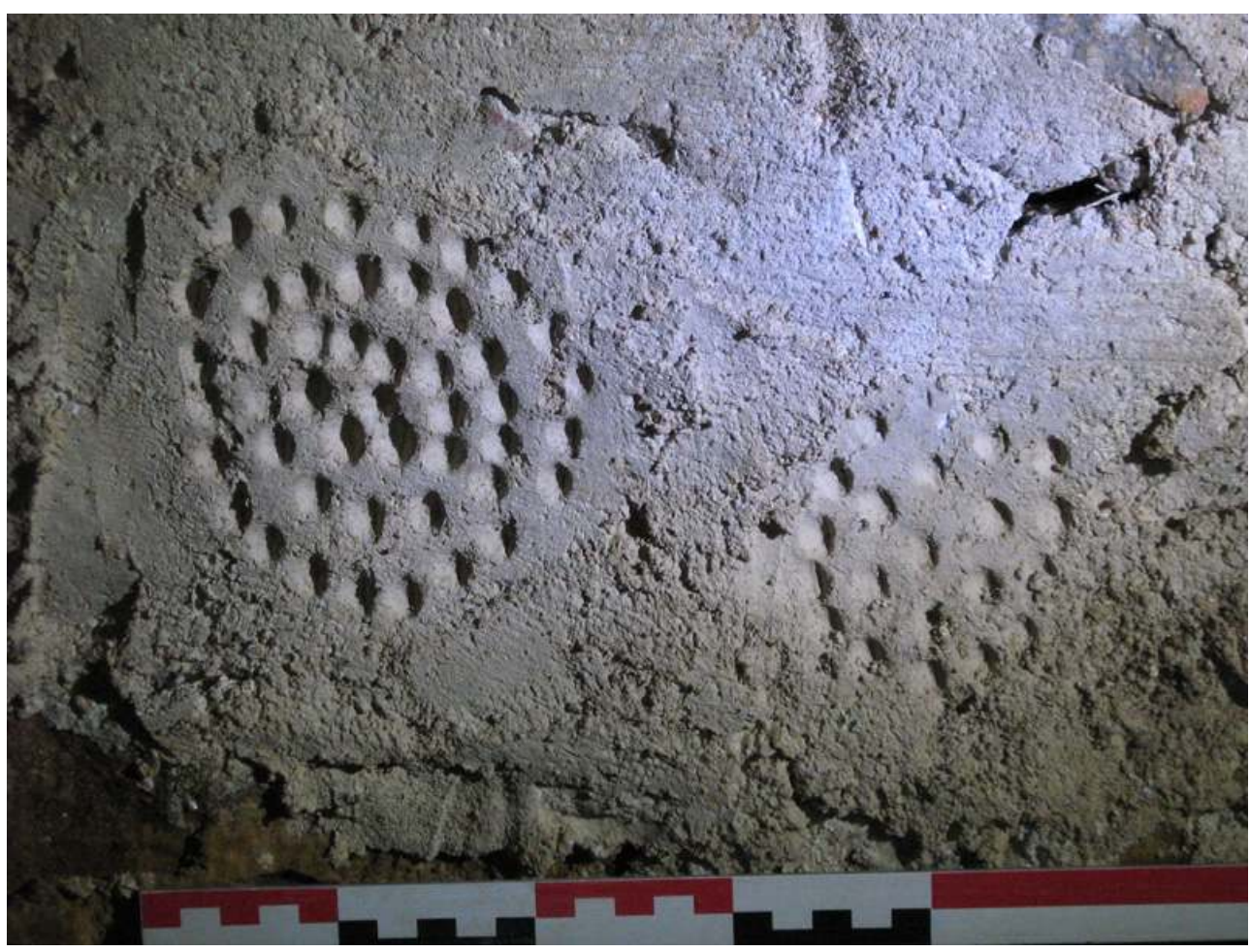

Auteur(s) : Bernot, Emmanuel (Service archéologique de la Ville de Lyon). Crédits : E. Bernot SAVL (2008)

\section{INDEX}

Thèmes : galerie, puits, souterrain

Index géographique : Rhône-Alpes, Rhône (69), Lyon (69123)

operation Fouille d'évaluation (EV)

Index chronologique : XVIe siècle apr. J.-C. 
AUTEUR

EMMANUEL BERNOT

SA ville de Lyon 